\title{
La opinión pública en la teoría de la Administración Pública
}

\section{Public opinion in Public Administration theory}

\author{
Juan Andrés Díaz Guerra \\ Universidad Complutense de Madrid (España) \\ jadiazgu@ucm.es
}

\section{NOTA BIOGRAFICA}

Licenciado en Ciencias Políticas y Sociología por la Universidad Complutense de Madrid (UCM). Gerente de Facultad. Anteriormente profesor de la UCM, URJC y UNED. Opinión pública y política de seguridad.

Manuel Tamayo Sáez

Universidad Rey Juan Carlos (España) manuel.tamayo@urjc.es

\section{NOTA BIOGRAFICA}

Doctor en Ciencias Políticas por la Universidad Complutense de Madrid (UCM). Profesor Contratado Doctor. Opinión pública y políticas públicas. Grupo de Investigación Diseño de Políticas: Transferencia e Innovación Social.

Ernesto Carrillo Barroso

Universidad Complutense de Madrid (España)

ORCID: https://orcid.org/0000-0002-9500-659X

ecarrillo@cps.ucm.es

\section{NOTA BIOGRAFICA}

Doctor en Sociología (UCM). Catedrático de Universidad. Instituto Complutense de Ciencia de la Administración. Grupo de Investigación Diseño de Políticas: Transferencia e Innovación Social. Opinión pública y políticas públicas.

\section{RESUMEN}

El propósito del artículo es reconstruir las aportaciones normativas que han realizado sobre la opinión pública algunos de los principales teóricos de la Administración, desde Wilson a Bozeman. Compartiendo con Lasswell la idea de que en democracia la relación entre opinión pública y políticas públicas debe ser bidireccional, las preguntas centrales desde esta perspectiva serían: ¿cuál debe ser el papel de la opinión pública en relación con la administración y las políticas públicas? y ¿hasta dónde es legítimo que desde las instituciones públicas se intente gestionar la opinión pública? Las respuestas ofrecidas podrían agruparse en una escala cuyo primer peldaño circunscribiría la opinión pública al papel de crítica autorizada que vigila el proceso de formación de las políticas, sin inmiscuirse en los asuntos cotidianos de la gestión; el segundo, estaría dirigido a sondear la opinión para definir estrategias y planes que garanticen el apoyo del público a las políticas, las regulaciones y los servicios; finalmente, el 
ciudadano, asumiría un papel más relevante y cotidiano, participando directamente e incluso eligiendo a los productores de los servicios públicos. La gestión de la opinión con fondos públicos podría considerarse legítima y necesaria, siendo de aplicación las herramientas de investigación de la opinión y de la comunicación institucional.

\title{
PALABRAS CLAVE
}

Marketing público; política pública; sondeos de opinión.

\begin{abstract}
This article reconstructs the normative contributions that some of the main theorists of the Administration have made about public opinion from Wilson to Bozeman. We agree with Lasswell that in a democracy the relationship between public opinion and public policies should be bidirectional. From this perspective, the central questions to be answered would be: what should be the role of public opinion in relation to public administration and policies? And to what extent is it legitimate for public institutions to try to manage public opinion? The answers offered could be grouped into a scale whose first step would circumscribe public opinion to the role of authoritative criticism that would oversee the process of policy formation without interfering in the day-to-day management issues; the second, would be aimed at probing opinion to define strategies and plans that guarantee public support for policies, regulations and services; finally, the citizen, would assume a more relevant and daily role participating directly and even choosing the producers of public services. The management of the opinion with public funds could be considered legitimate and necessary, with the application of opinion research and institutional communication tools.
\end{abstract}

\section{KEYWORDS}

Marketing government; opinion polls; public policy.

\section{SUMARIO}

INTRODUCCIÓN. 1. LAS REFERENCIAS A LA OPINIÓN PÚBLICA EN LA TEORÍA DE LAADMINISTRACIÓN PÚBLICA. 1.1. WILSON Y LA OPINIÓN PÚBLICA COMO CRÍTICAAUTORIZADA QUE VIGILA LAS GRANDES FUERZAS DE LA FORMACIÓN DE LAS POLÍTICAS PÚBLICAS. 1.2. WHITE Y LA FUNCIÓN DE INTERPRETAR Y DEFENDER LAS POLÍTICAS PÚBLICAS Y EL PROBLEMA DE LA GESTIÓN DE LA OPINIÓN PÚBLICA DESDE LOS ORGANISMOS OFICIALES. 1.3. LASSWELL Y EL APOYO DE LA OPINIÓN PÚBLICAA LA DEMOCRACIA: EL INTERCAMBIO ABIERTO ENTRE OPINIÓN PÚBLICA Y POLÍTICA PÚBLICA COMO SEÑA DE IDENTIDAD DE LAS DEMOCRACIAS. 1.4. LA OPINIÓN PÚBLICA Y EL APOYO A LAS ORGANIZACIONES GUBERNAMENTALES EN SIMON, SMITHBURG Y THOMPSON: LAS TÁCTICAS PARA OBTENER EL APOYO Y LOS SONDEOS DE OPINIÓN COMO INSTRUMENTO PARA LA PLANIFICACIÓN ADMINISTRATIVA. 1.5. LAS PREFERENCIAS INDIVIDUALES Y LA ADMINISTRACIÓN DEMOCRÁTICA DE OSTROM. 1.6. LA NUEVA ADMINISTRACIÓN PÚBLICA Y LA GOBERNANZA RECEPTIVA. 1.7. LA SATISFACCIÓN DEL CONSUMIDOR Y EL NUEVO MANAGEMENT PÚBLICO. 1.8. LA CREACIÓN DE VALOR PÚBLICO: LOS DESEOS DE LOS CIUDADANOS Y SUS REPRESENTANTES Y EL PAPEL DEL MARKETING PÚBLICO PARA INCREMENTAR EL APOYO POLÍTICO A LAS POLÍTICAS. 1.9. BOZEMAN: LA AUTORIDAD POLÍTICA DE LAS POLÍTICAS, LA IDENTIFICACIÓN DE LOS VALORES PÚBLICOS DE LAS POLÍTICAS Y LOS FALLOS PÚBLICOS. 2. CONSIDERACIONES NORMATIVAS SOBRE LA RELACIÓN ENTRE OPINIÓN PÚBLICA Y ADMINISTRACIÓN PÚBLICA. 2.1. ¿CUÁL DEBE SER EL ALCANCE DE LA INFLUENCIA DE LA OPINIÓN PÚBLICA SOBRE LA ADMINISTRACIÓN Y LAS POLÍTICAS PÚBLICAS? 2.2. ¿HASTA DÓNDE ES LEGÍTIMO QUE EL GOBIERNO ACTÚE SOBRE LA OPINIÓN PÚBLICA?. 2.2.1. La interpretación y defensa de las políticas. 2.2.2. La gestión de la opinión pública mediante recursos públicos. CONCLUSIONES. REFERENCIAS BIBLIOGRÁFICAS.

\section{INTRODUCCIÓN}

La cuestión de la opinión pública, y más concretamente el papel que esta deba desempeñar en la administración de un sistema político democrático, es un tema sobre el que han reflexionado los estudiosos de la Ciencia de la Administración. Nunca ha constituido un tema central de la disciplina, pero las alusiones a la opinión pública son recurrentes en los textos clásicos de Administración y Política Pública, en particular 
en Wilson, White, Lasswell o Simon. Con el paso del tiempo las reflexiones sobre este tema se hacen más esporádicas e indirectas, pero ello no impide que su observación pueda abordarse desde una perspectiva administrativa. El propósito de este artículo es, precisamente, reconstruir las aportaciones que sobre la opinión pública han realizado algunos de los principales teóricos de la Administración y ofrecer un estado de la cuestión al respecto.

Desde sus orígenes los estudios de Administración Pública han sido sensibles al tema de la opinión pública planteándose preguntas normativas -incluidos sus detalles más prácticos- que permitieran orientar el comportamiento del directivo público: ¿Qué papel debe tener la opinión pública en la conducción de la Administración? ¿A qué función del Estado debe corresponder el arte de influir en la opinión, a la Política o a la Administración? ¿Es legítimo que un organismo público intente gestionar la opinión pública? ¿Cómo deben ser las relaciones entre opinión pública y política pública? ¿A qué tipo de conocimientos profesionales recurrir para gestionar la opinión pública? ¿Qué utilidad pueden tener los sondeos de opinión y la investigación de mercados en la formulación de estrategias y la planificación administrativa? ¿Qué opiniones nos deben interesar, las de los ciudadanos y sus representantes o las de los consumidores directos de los servicios? ¿En qué medida los estudios de opinión pública nos informan sobre los valores públicos? ¿Es legítimo el uso del marketing en el sector público? ¿Qué limitaciones deben existir en el manejo de las herramientas de promoción en la administración?

Como se verá seguidamente, todas estas preguntas han sido abordadas por la teoría de la Administración desde sus orígenes hasta nuestros días y a ellas se intentará dar respuesta de forma sumaria en este trabajo. Para ello se ha optado por recopilar cronológicamente las principales referencias concernientes al tema de la opinión pública en los textos clásicos y en otros más recientes, desde Wilson a Bozeman, recogiendo las aportaciones que en diferentes épocas y desde distintas escuelas se han ofrecido para esta cuestión ${ }^{1}$. Nos hemos centrado en las reflexiones de carácter normativo y aplicado de mayor interés para los directivos públicos, dejando al margen los estudios que consideran el papel que de hecho ejerce la opinión pública en la administración y la formación de las políticas. Además de la exposición cronológica, en la segunda parte del artículo se han conjugado todas esas reflexiones, dispersas a modo de teselas, en un mosaico que dé respuesta integrada a las preguntas planteadas en esta introducción.

\section{LAS REFERENCIAS A LA OPINIÓN PÚBLICA EN LA TEORÍA DE LA ADMINISTRACIÓN PÚBLICA}

\subsection{Wilson y la opinión pública como crítica autorizada que vigila las grandes fuerzas de la formación de las políticas públicas}

Wilson, en el que es considerado el trabajo seminal de este campo de estudios, "The Study of Administration", publicado en Political Science Quarterly en 1887, dedica varios párrafos a tratar el asunto. Este autor se plantea como uno de los problemas de la nueva disciplina de la Ciencia de la Administración el de abordar la pregunta de «¿qué parte debe tener la opinión pública en la conducción de la administración?». Su respuesta fue que «la opinión pública debe desempeñar el papel de crítica autorizada» (Wilson, 1887, pág. 214). Más adelante señala lo siguiente:

«El problema consiste en hacer que la opinión pública sea eficiente, sin el dolor de que sea entrometida. Ejercida directamente, en la supervisión de los detalles cotidianos y en la elección de los medios diarios del gobierno, la crítica pública es, desde luego, un verdadero fastidio, como un rústico al que se le confiara una maquinaria delicada. Mas, para vigilar las grandes fuerzas de la formación de la política [policy formation], tanto en la política [politics] como en la administración, la crítica pública es por completo segura y benéfica, a la par que indispensable. Permitamos que el estudio administrativo encuentre los mejores medios de otorgar este control a la crítica pública y ahuyentémosla de cualquier otra interferencia» (Wilson, 1987, pág. 215).

1 El lector encontrará lagunas importantes como Weber; sin embargo, las reflexiones de este autor sobre la opinión pública son escasas y no están vinculadas a la parte que más nos puede interesar sobre la sociología de la dominación y el tipo ideal racional legal con cuadro administrativo burocrático. 
No deja de resultar significativo que en un breve artículo en que intenta poner las bases de la Ciencia de la Administración dedique atención a la cuestión de la opinión pública. Al plantear este asunto, Wilson llama la atención sobre las características y complejidades de la administración en los sistemas políticos democráticos. Concretamente, cabría argumentar que considera más complejo administrar en una democracia que en otros sistemas políticos, debido precisamente al papel desempeñado por la opinión pública en la administración democrática.

En el terreno práctico, ya como presidente de los Estados Unidos, otorgó también especial importancia a la "gestión» de la opinión pública, desarrollando un considerable esfuerzo por comunicar sus políticas. Según White, fue Wilson quien introdujo las ruedas de prensa en Washington (White, 1955, pág. 200), en las que el propio presidente se dirigía directamente a la nación a través de las preguntas y respuestas a los corresponsales. Desde luego, era un presidente muy interesado en la opinión pública y tuvo entre sus asesores al joven Lippman. Según describe este en su libro Public Opinion (1922, 2014), la Administración de Wilson llevó a cabo para la época un gigantesco esfuerzo de comunicación directa a la ciudadanía durante la Primera Guerra Mundial, una experiencia en la que se basaron los esfuerzos de propaganda y relaciones con el público que desarrollarán posteriormente tanto las empresas como la administración (Lasswell, 1941).

\subsection{White y la función de interpretar y defender las políticas públicas y el problema de la gestión de la opinión pública desde los organismos oficiales}

La cuestión de la gestión de la opinión pública ha sido abordada por otros clásicos de la Ciencia de la Administración, planteándose el problema de si es legítimo o no intentar gestionar la opinión pública y, en su caso, a quién compete hacerlo y a través de qué medios. Así, Goodnow parte de la distinción entre dos tipos de funciones del Estado [government], Política y Administración: «La Política se relaciona con las políticas o las expresiones de la voluntad del Estado. La Administración con la ejecución de esas políticas» (Goodnow, 1900, pág. 19), ubicando al arte de influir en la opinión pública en la esfera política.

Siguiendo esa misma estela de la dicotomía entre Política y Administración, White -a quien se atribuye el primer manual de Administración Pública, Introduction to the Study of Public Administration (1926, 1955)asigna a la «función de línea en el nivel político» diversas funciones -distintas de las del nivel administrativo-, entre las que se encuentra la de «interpretar y defender la política [policy] y sus operaciones» (White, 1955).

Esta sería una función propia del líder del ejecutivo, de los responsables de los departamentos ministeriales y de sus gabinetes políticos, «resultando particularmente inadecuada en el caso de los funcionarios de carrera» (White, 1955). Las comparecencias y debates parlamentarios en los que participan el presidente y sus ministros, los discursos de investidura y del estado de la nación, las ruedas de prensa regulares tras los consejos de ministros, las declaraciones del presidente y de los miembros de su gabinete ante los medios de comunicación o en actos públicos recogidos por la prensa, serían las fórmulas a través de las que se lleva a cabo esta función. A ello podrían añadirse los contactos con los grupos de interés y las campañas electorales.

Estas actividades son consideradas legítimas en cualquier democracia; sin embargo, algunos pasos más allá en esta función pueden resultar más controvertidos planteándose:

«el problema de la gestión de la opinión mediante medios oficiales. Es importante para las autoridades del gobierno [government officials] conocer el estado de la opinión sobre su trabajo y sus organizaciones. Es inteligente, por parte de ellos, tratar de cultivar una opinión pública favorable y reducir la indiferencia o la hostilidad. Hasta dónde tienen derecho a llegar en la formación y administración de la opinión pública, es materia sobre la cual hay grandes diferencias de criterio» (White, 1955).

White menciona distintos instrumentos empleados regularmente por los organismos públicos para sondear la opinión, entre los que destaca las encuestas sobre la opinión pública porque «proporcionan datos sobre cuestiones importantes de política pública», los referenda sobre temas específicos, el análisis de los periódicos y otro material impreso y el establecimiento de «puestos específicos de escucha para informarse».

Asimismo, añade que «es parte de la prudencia de todo organismo oficial, no simplemente saber cómo lo valora el público, sino también cultivar una disposición amistosa en quienes pueden afectar a su futuro». Con esta segunda apreciación se adentra en un tema más espinoso, cual es el de la formación y la gestión de la opinión mediante recursos públicos, ya sea a través de la publicidad o de cualquier otra fórmula de 
promoción y comunicación institucional de las políticas públicas. Las posiciones varían desde quienes lo consideran ilegítimo o propio de sistemas políticos autoritarios que intentan imponer como única opinión la del propio gobierno; hasta quienes lo ven no solo como algo legítimo, sino también necesario. De hecho, es común que en los sistemas democráticos se establezcan regulaciones para evitar posibles abusos, en especial con la publicidad institucional, si bien, tanto en la época de White como en la actualidad suelen ser de escasa utilidad.

A modo de resumen White concluye:

«Baste decir que en el mundo contemporáneo las autoridades necesitan aquiescencia, que, por lo tanto, están interesados en el estado de la opinión acerca de su trabajo, que pueden hacer, con toda propiedad, gestiones para pulsar la opinión, que tienen un interés personal y legítimo en ilustrarla, ofreciéndole información exacta sobre hechos, y que, en muchos casos, dan pasos positivos para contribuir a formar una opinión favorable al programa que ellos respaldan. Estas actividades figuran entre los aspectos básicos de la función de línea.».

\subsection{Lasswell y el apoyo de la opinión pública a la democracia: el intercambio abierto entre opinión pública y política pública como seña de identidad de las democracias}

Lasswell, impulsor de los estudios de política pública, dedicó también especial atención a la cuestión de la opinión pública. En su libro Democracy Through Public Opinion (1941), hace depender la supervivencia de la democracia del apoyo proporcionado por la opinión pública a los fines y a los medios del gobierno democrático. Los fines, señala, tienen un carácter permanente y consisten en la práctica de la justicia mediante el gobierno de la mayoría. Los medios, en cambio, son provisionales, ajustados a las necesidades y oportunidades del momento, y se concretan en demandas de políticas públicas que han de ser congruentes con los fines permanentes. Esta distinción entre fines y medios hace reparar en la necesidad de contar con una opinión pública que apoye tanto los procedimientos democráticos a través de los cuales se gobierna como las demandas sustantivas respecto de los temas centrales de política pública de cada momento histórico.

Cuando existe un alto grado de correspondencia entre los actos del gobierno y la opinión mayoritaria durante un cierto periodo de tiempo se produciría el «gobierno por la opinión pública». Aunque el ajuste entre política pública y opinión sea la situación óptima no siempre se produce, pudiendo existir periodos en que las políticas no respondan a las demandas sustantivas de los ciudadanos.

Para Lasswell, la relación entre el gobierno y la opinión pública en los sistemas políticos democráticos debe ser bidireccional. El gobierno actúa sobre la opinión y esta debe hacerlo a su vez sobre el gobierno, siendo «el intercambio abierto entre opinión y política [pública] la seña de identidad del gobierno del pueblo» (Lasswell, 1941). El que la comunicación sea bidireccional es característico de las democracias, puesto que según señala este autor, los regímenes autoritarios intentan influir en la opinión pública, pero en cambio evitan recibir la influencia de la opinión, siendo la comunicación exclusivamente de una sola dirección.

Esta relación entre opinión pública y política pública da pie a que Lasswell plantee numerosas derivadas del problema, como son entre otras: el de los posibles efectos de la congruencia o no entre las políticas públicas adoptadas por los gobiernos y las demandadas por los ciudadanos; la necesidad de que las sociedades dispongan de adecuados niveles de información en los que basar sus opiniones; el papel desempeñado por los científicos y periodistas para generar esa información -unos, descubriendo la verdad, y los otros clarificando los resultados de la investigación científica, haciéndolos accesibles y despertando el interés del público por estas cuestiones-; los riesgos que puede entrañar para la democracia que las noticias que leen, escuchan y ven los ciudadanos estén manipuladas por propagandistas, que puedan operar incluso en contra de la democracia aprovechando las libertades que esta ofrece; el papel fundamental que pueden desempeñar los expertos en relaciones con el público, como asesores de quienes adoptan decisiones de política pública, una especie de alter ego del decisor que le ayuda a adaptar las políticas al ambiente en el que han de operar, proyectándolas hacia el futuro y promocionándolas a través de la información, las relaciones con los medios, la publicidad y la propaganda.

Esa relación bidireccional entre opinión pública y política pública, propia de gobiernos receptivos a las demandas de los ciudadanos y de una ciudadanía bien informada que reacciona a las actuaciones de los gobiernos, coloca a la opinión pública sobre las políticas públicas en un lugar central de los sistemas políticos democráticos. Al mismo tiempo, para que esta opinión pública opere a favor del interés general destaca el papel desempeñado por la generación de conocimiento científico sobre los problemas públicos y los medios 
de comunicación en su difusión, así como las tareas de promoción y comunicación institucional que deben desarrollar los gobiernos para que los ciudadanos puedan hacerse una sólida opinión sobre las políticas públicas.

\subsection{La opinión pública y el apoyo a las organizaciones gubernamentales en Simon, Smithburg y Thompson: las tácticas para obtener el apoyo y los sondeos de opinión como instrumento para la planificación administrativa}

Cuando Simon, Smithburg y Thomson escribieron Public Administration se propusieron elaborar un libro que reflejara el funcionamiento real del gobierno. A lo largo del texto se recogen reflexiones sobre la opinión pública, que intentaremos compilar y resumir, y que efectivamente permiten reconocer comportamientos reales, al menos de gobiernos receptivos a las necesidades y las demandas de los ciudadanos así como conscientes de la importancia de la opinión pública en una administración democrática.

Los autores consideran a las organizaciones gubernamentales como sistemas sociales que se mantienen en un equilibrio dinámico. En ese sentido, tienen que adaptarse al mundo que les rodea estableciendo relaciones con diversos «participantes» externos. Esas relaciones tienen un carácter esencialmente político y en ellas se producen intercambios entre la organización y los participantes. Estos reciben de la organización incentivos a cambio de las contribuciones que proporcionan a la organización. Entre los participantes con los que se relacionan las organizaciones gubernamentales se encuentran: la asamblea legislativa, el líder ejecutivo, otras organizaciones gubernamentales, los grupos a los que regula o sirve la organización (los grupos de clientes) y el público general.

Bajo el concepto de clientes se alude al grupo de la sociedad que está más directamente interesado en los programas de la organización. Si los clientes forman un grupo amplio, con intereses comunes y bien organizado ejercerán notable impacto sobre la asamblea legislativa; y su apoyo, o al menos su aquiescencia, será importante cuando no necesario para la supervivencia de la agencia administrativa en cuestión. Por otra parte, en el caso de que la organización al planificar o llevar a cabo sus programas requiera de la cooperación de segmentos del público o incluso del público en su totalidad, será importante también el apoyo de los grupos externos, entre otras cosas porque aquellos públicos a los que se regula deberán aceptar los programas, o al menos acceder a ellos.

Generalmente los grupos de presión ejercen más influencia que el público general, pues la opinión pública suele ser más vaga y puede carecer de coherencia general. Sin embargo, en la medida en que el público general vota puede convertirse en una fuente de apoyo o rechazo de la organización administrativa, de modo que la opinión pública deberá ser tenida en cuenta por el partido que está en el poder. En los casos en que los sondeos de opinión muestren una opinión de la mayoría y una opinión entusiasta de la gente, podrá contrarrestarse a los grupos de presión y podrán contribuir a abolir o modificar de forma sustancial cualquier programa gubernamental.

Los autores analizan también las tácticas de supervivencia que adoptan las organizaciones administrativas para buscar el apoyo externo. En lo que respecta al apoyo público, las agencias administrativas deben asegurarse de que los ciudadanos poseen información adecuada de sus programas, ya sea porque les proporcionen servicios o porque regulen sus comportamientos. Si el público no conoce los servicios los funcionarios fracasarán en sus intentos de ofrecerlos. Si no saben lo que tienen que hacer como consecuencia de una regulación no lo harán. Si no están de acuerdo con lo que se supone que tienen que hacer, muchos probablemente tampoco lo harán. Por tanto, las agencias administrativas tienen que dedicar un importante esfuerzo a actividades de educación del público. Para ello diseminan grandes volúmenes de información obtenida mediante la investigación; informan constantemente a los públicos de los servicios disponibles; e informan al público (o a partes de él) de sus obligaciones derivadas de las regulaciones.

Ello conlleva el desarrollo de actividades de información pública y propaganda -incluida la publicidad pagada y no pagada- y actividades de relaciones con el público en general. Con frecuencia, estas actividades van más allá de la mera información al público para pasar a la «venta» de sus programas, lo que puede generar reacciones adversas de las asambleas legislativas y de los grupos de interés hostiles a la agencia o a sus programas. Por ello se suelen producir regulaciones que limitan estas actividades. No obstante, es difícil distinguir las relaciones con el público legítimas e ilegítimas, con lo cual el impacto de estas regulaciones es limitado.

Entre las técnicas de publicidad no pagada a las que recurren las organizaciones gubernamentales los autores mencionan a los gabinetes de prensa, la inclusión en programas de radio y de películas de una ima- 
gen favorable de la agencia, las declaraciones públicas de los administradores - planteándose la cuestión del control de estas declaraciones y la necesidad de «negros» que escriban los discursos e intenten prever las reacciones del público-, las filtraciones a la prensa y la formación del personal que está en contacto directo con el público. Es importante, recuerdan, que la propaganda se base en hechos.

Más útil que la propaganda es una buena planificación del programa y se mencionan expresamente las encuestas de opinión: mediante ellas se puede conocer lo que le gusta a la gente y lo que no y el por qué, y con esta información planificar. Entre otros extremos, son útiles para conocer el grado de aceptación de los programas y las regulaciones y, lo que es más interesante, para hacer un diagnóstico de la disconformidad, conociendo el grado de disconformidad de diferentes segmentos de población y en distintas partes del país y, quizá, las razones de la disconformidad. Esta información puede ayudar a decidir si intervenir o no y, en caso afirmativo, qué hacer, así como a las tareas de planificación, educación y propaganda.

Los estudios de opinión pública formarían parte de los sistemas de información y comunicación de las organizaciones, concretamente de las unidades de inteligencia encargadas de recabar la información que necesita la organización; más en particular, de las unidades de inteligencia externa, las cuales recopilan, analizan y distribuyen información que no forma parte de las operaciones cotidianas de la organización. La función de inteligencia del alto grupo ejecutivo consiste en recabar e interpretar la información sobre las reacciones de los grupos externos políticamente importantes para los programas de la organización, incluidos los clientes de la organización y el público en general, recopilando información sobre los impactos de los programas y sobre sus reacciones. Desde luego, una organización que planifique tomando en cuenta este tipo de información no solo tendrá más éxito en sus programas, sino que políticamente se encontrara en mejor posición para la lidiar con los restantes actores políticos.

En síntesis, ya no es solo la democracia la que puede depender de la opinión pública, sino también las organizaciones gubernamentales concretas, las cuales para lidiar con su entorno político y para planificar y gestionar sus programas necesitan investigar el estado de la opinión pública y recurrir a otros instrumentos de comunicación que ayuden a los gestores públicos en la prestación de servicios y para conseguir que se cumplan sus regulaciones.

\subsection{Las preferencias individuales y la Administración Democrática de Ostrom}

Las distintas Teorías de la Administración Pública que se desarrollan durante las décadas de 1970 y 1980 tienen en común la crítica a la Administración Pública clásica, proponiendo «nuevos» modelos antiburocráticos. Así sucede con la Administración democrática de Ostrom, las Nueva Administración Pública y el movimiento del Nuevo Management Público. Estas tres corrientes comparten también una especial preocupación por uno de los valores democráticos clave, el de la receptividad, formulando propuestas a favor de una Administración receptiva, ya sea -respectivamente- hacia las preferencias de los individuos, las de los ciudadanos o las de sus clientes, según cada una de las teorías arriba señaladas.

En su libro The Intellectual Crisis in American Public Administration (1971, 1989) Ostrom propone un cambio en el objeto de estudio de la Administración Pública que gire en torno al concepto de bienes públicos, frente al de burocracia en el que se había basado la Administración Pública tradicional. Ello es especialmente relevante pues:

«Cuando se considera que el problema principal de la administración pública es la provisión de bienes y servicios públicos, podemos disponer de formas alternativas de organización distintas de la ampliación y perfeccionamiento de las estructuras burocráticas. Las estructuras burocráticas son necesarias, pero no suficientes para una economía de los servicios públicos receptiva y productiva» (Ostrom, 1971, 1989, pág. 17).

Al dar este giro conceptual se abre la puerta a muy diversas formas de intervención pública, en el entendido de que la fórmula elegida en cada caso concreto tendrá consecuencias respecto de los outputs de bienes y servicios públicos, tanto en términos de eficiencia como en cuanto a otros criterios con los que se pueda evaluar el rendimiento del mecanismo institucional elegido. De este modo, para los estudios de Administración Pública la pregunta fundamental que tratan de responder es "¿en qué situaciones es más eficiente y responde mejor a las preferencias de los individuos en distintos contextos sociales el recurso a fórmulas burocráticas u otros tipos de mecanismos organizativos?». El criterio de receptividad a las preferencias de los individuos pasa así a ocupar un lugar central, compartiendo protagonismo con el más tradicional de eficiencia. 
Asimismo, cuestiona la centralización y la capacidad de los grandes sistemas administrativos «para dar respuesta a las diversas preferencias existentes entre los ciudadanos respecto de los bienes y servicios públicos», su flexibilidad para adaptarse a las condiciones del entorno e incluso su eficiencia. Al mismo tiempo, propone la fragmentación de la autoridad entre diversos centros de decisión, el desarrollo de múltiples jurisdicciones con diferentes escalas y el recurso a fórmulas de gobierno multiorganizativo.

Lo importante a nuestros efectos es que la propuesta de Ostrom no sólo pone el énfasis en generar una provisión y producción más eficiente de servicios, sino también en que esta sea más democrática. La preocupación por la receptividad del gobierno a las preferencias individuales está, como vimos, en el centro de su razonamiento. De hecho, los criterios que deben servir para elegir unidades de provisión priorizan que permitan abordar los problemas revelados por las preferencias de los ciudadanos, la equivalencia fiscal y la rendición de cuentas. Así, la fragmentación de la división municipal está justificada por la necesidad de que los individuos puedan escoger su lugar de residencia -esto es, que puedan votar con los pies- tomando en consideración el paquete de impuestos y servicios más acorde con sus necesidades dentro de los ofrecidos por los distintos gobiernos locales. El propio concepto de eficiencia en las unidades de provisión y de producción es diferente: una unidad de provisión será eficiente en la medida en que revele y satisfaga adecuadamente las preferencias de los ciudadanos, mientras que la eficiencia de una unidad de producción radica en transformar recursos en productos al menor coste posible. Ello explica que un gobierno pueda adoptar las decisiones de provisión y que, al mismo tiempo, en lugar de producir el servicio con una burocracia pública bajo su mando directo, lo haga contratando a una empresa, suscribiendo convenios con otros gobiernos o con organizaciones no gubernamentales o mediante cualquier otra fórmula.

Aunque no hemos encontrado en Ostrom ninguna referencia a la opinión pública o a la utilización de sondeos para conocer las preferencias - posiblemente porque su modelo, basado en el individualismo metodológico, le lleve a soslayar estas cuestiones-, sin embargo, al haber otorgado un énfasis especial a la cuestión de la receptividad, resulta conveniente tomarlo en consideración en esta revisión de las teorías de la Administración Pública.

\subsection{La Nueva Administración Pública y la Gobernanza receptiva}

Quizá la principal contribución de la Nueva Administración Pública haya consistido en incorporar el criterio de la equidad social a los objetivos clásicos de eficiencia y economía de la Administración Pública convencional, planteando la pregunta de si «¿este servicio mejora la equidad social?» (Fredericson, 1980). Cuando Frederickson emplea el concepto de equidad social lo hace para referirse a una amalgama amplia de valores, diseños organizativos y estilos de gestión que pretende promocionar, entre ellos el valor de la receptividad. Así, en el libro New Public Administration (1980) señala, entre muchos otros atributos, que «la equidad social hace hincapié en la capacidad de respuesta a las necesidades de los ciudadanos en lugar de a las necesidades de las organizaciones públicas».

Asimismo, atribuye a los ciudadanos un papel activo en su relación con la administración. Lejos de los planteamientos jerárquicos y autoritarios de la administración tradicional propone la participación de los clientes como uno de los conceptos antiburocráticos que caracterizan a la Nueva Administración Pública. Frente a la Administración Pública clásica, que está orientada hacia las instituciones, la Nueva Administración Pública está orientada hacia el impacto sobre sus clientes. Le interesa en particular el impacto que ejerce la organización sobre sus clientes y viceversa, la influencia de estos en la organización. El compromiso con la receptividad y la equidad social lleva a la Nueva Administración Pública a promover la participación en el proceso de elaboración de las políticas públicas.

Considera a la administración pública como «el vehículo para implementar los valores o preferencias de individuos, grupos, clases sociales o sociedades enteras». Muchos de los valores que promocionan las organizaciones gubernamentales con frecuencia son incompatibles entre sí, siendo necesario establecer alguna priorización. La Nueva Administración Pública propone priorizar nuevos valores frente a los tradicionales, sin renunciar por ello a estos últimos:

«Los valores tradicionales de la administración pública -economía, eficiencia, eficacia, receptividad hacia las autoridades políticas, responsabilidad- nos han servido bien. Pero las experiencias de la última década indican que otros valores -no necesariamente nuevos- son importantes para cualquier ética de servicio público. Ciertamente, la receptividad hacia los ciudadanos, la participación de los trabajadores y de los ciudadanos en el proceso de decisión, la 
distribución equitativa de los servicios públicos, la provisión de una variedad de opciones entre las que puedan elegir los ciudadanos y la responsabilidad administrativa por la eficacia de los programas son valores que se adaptan a nuestro tiempo. Si existe, o incluso debería haber, una ética de servicio público, estos valores son tan convincentes como lo son los compromisos mejor entendidos con los valores gerenciales» (Fredericson, 1980).

Cuando Frederickson piensa en qué mecanismos estructurales e instrumentos de gestión pueden coadyuvar a la promoción de los valores que propone priorizar, en ningún momento está considerando los sondeos de opinión u otras herramientas de conocimiento de la opinión pública que puedan utilizarse para hacer más receptiva y participativa a la administración. Está pensando, entre otras cuestiones, en consejos vecinales y otros procedimientos que permitan controlar a los «street level»; en el establecimiento de rutinas en las que los gestores interactúen con sus clientes y sus empleados; en potenciar la receptividad no solo hacia los políticos sino también hacia los grupos de interés y las minorías desorganizadas; en el derecho a que los trabajadores y los ciudadanos participen en los procesos de adopción de decisiones que les afectan directamente a sus vidas; etc.

Es decir, está pensando en la primera generación de instrumentos de participación ciudadana diseñados para grupos de ciudadanos organizados -como los consejos de barrio, sectoriales, escolares, etc.-y en promover un tipo de democracia más avanzado, donde los ciudadanos no se limiten únicamente a remplazar a los gobernantes a través de las elecciones, sino que se impliquen de forma directa dentro de todo el proceso de elaboración de las políticas. Sin embargo, lo que nos importa más de su enfoque a efectos de este artículo es la prioridad que otorga a los valores de receptividad y participación.

\subsection{La satisfacción del consumidor y el Nuevo Management Público}

Una de las características del Nuevo Management Público reside en hacer «hincapié en la calidad del servicio y en la orientación al consumidor» (Pollitt, 2003). El ciudadano pasa a ser considerado como un «cliente» al que se ofrecen servicios diseñados a su mayor conveniencia, «prestando más atención a cómo satisfacer las demandas de los ciudadanos y a proporcionar servicios a través de organizaciones distintas de las burocracias públicas tradicionales» (Hood, 2002).

Según Aucoin (1990) estas ideas, junto a muchas otras de las formuladas por el movimiento del Nuevo Management Público, provienen de los estudios de Administración de Empresas, en particular del libro de Peters y Waterman In Search of Excellence: Lessons from America's Best-run Companies. En este texto se identifica la «proximidad al cliente» como uno de los ocho atributos que caracterizan a las empresas sobresalientes:

«Estas empresas aprenden de las personas a quienes sirven. Ofrecen calidad sin igual, servicio y fiabilidad...consiguen diferenciar entre sí los productos más análogos. Muchas de las empresas tuvieron sus mejores ideas de los clientes, y eso se consigue a fuerza de escuchar con atención y de modo regular» (Peters y Waterman, 1992).

Se trataría de empresas orientadas hacia el cliente, al que escuchan mediante procedimientos formales e informales -incluida la investigación de mercados-, que buscan sus huecos de mercado en que puedan destacar frente a sus competidores, que segmentan a sus consumidores en grupos para proporcionarles servicios a su medida, que están preocupadas por la calidad del servicio y que miden la satisfacción de sus clientes (Peters y Waterman, 1982, 1992).

En uno de los textos más conocidos a favor del Nuevo Management Público, Reinventing Government: How the Entrepreneurial Spirit is Transforming the Public Sector de Osborne y Gaebler, se traducen al sector público estos planteamientos empresariales. Así, proponen un «customer-driven government» preocupado por las necesidades del cliente y no de la burocracia. Esta última, según su crítica, está más orientada hacia los grupos de interés que hacia los ciudadanos; ignora a sus clientes, pues no se financia directamente de ellos; es arrogante con sus consumidores tratándolos como públicos cautivos, cuando una proporción muy notable de la población está muy cualificada y demanda que se la trate como cliente; y ofrece servicios estandarizados sin posibilidad de elección, a pesar de la heterogeneidad de la población.

Para Osborne y Gaebler la alternativa a la burocracia pasa porque las instituciones públicas se financien como las empresas, de modo que el ciudadano tome las riendas y pueda elegir. Ese será el camino más directo para que los gobiernos atiendan las necesidades de sus clientes. Para ello proponen el abanico de 
mecanismos institucionales que había identificado la teoría de la elección pública, distintos de las burocracias tradicionales, para permitir a los consumidores elegir a su proveedor ${ }^{2}$. Paralelamente, sugieren recurrir a los métodos de la calidad total, ofreciendo al ciudadano servicios amigables, claros e integrales. Por último, reclaman de las organizaciones públicas que estén próximas al cliente, escuchándole mediante diferentes herramientas en las que se conjugan, entre otras, técnicas de investigación de mercado, métodos de participación ciudadana y sistemas de control de calidad: encuestas dirigidas a distintos universos (consumidores de servicios, antiguos usuarios, residentes de ciudades o comunidades), grupos de discusión, entrevistas en profundidad, consejos de usuarios y consejos vecinales, oficinas de atención al cliente y el establecimiento de la figura defensor del pueblo o del usuario, atención directa al público a cargo de altos funcionarios de forma limitada pero con carácter periódico para entender mejor a los clientes, recepción y respuesta de correo electrónico por parte de los servicios de la administración, cursos de atención al público para funcionarios, cartas de servicios, inspecciones de servicios -recurriendo también al «comprador misterioso»-, buzones de quejas y sugerencias -incluidos números de teléfono de llamada gratuita para informar sobre atención inadecuada por parte de funcionarios-, comisiones de seguimiento de reclamaciones y traslado de informes de quejas a los empleados públicos afectados (Osborne y Gaebler, 1992).

\subsection{La creación de valor público: los deseos de los ciudadanos y sus representantes y el papel del marketing público para incrementar el apoyo político a las políticas}

Moor, en su libro Creating Public Value: Strategic Management in Government, también reflexiona sobre estas cuestiones. Para este autor «el objetivo del trabajo gerencial en el sector público es crear valor público, al igual que el objetivo del trabajo gerencial en el sector privado es crear valor privado» (Moor, 1995). Sin embargo, llegar a determinar en qué consiste el valor público no es una tarea sencilla.

Según Moor el valor está enraizado en los deseos y percepciones de los individuos. Algunos de estos deseos los proporciona el mercado, otros en cambio no. A los gestores públicos les interesan únicamente aquellas aspiraciones de los ciudadanos respecto de bienes y servicios proporcionados por el Estado y que se expresan a través de las instituciones del gobierno representativo. En este sentido, los gestores públicos crean valor en la medida en que satisfacen las preferencias y deseos políticos de los ciudadanos. Los mercados a los que se dirigen son de dos tipos diferentes: los clientes o beneficiarios directos de los bienes y servicios públicos, y los ciudadanos y sus representantes -sería algo parecido a lo que sucede a los gestores de empresas que deben satisfacer al consumidor y a los accionistas-. Los «accionistas» en el sector público -los ciudadanos y sus representantes- demandarán no solo una producción y distribución de bienes eficiente, sino también justa. Las aspiraciones de los ciudadanos son cambiantes, lo cual obliga a los gestores a experimentar e innovar. Finalmente, señala que:

«Los ciudadanos y sus representantes (en contraposición a los clientes y beneficiarios de los programas) lo que "compran" a los administradores públicos es un relato de la empresa pública, una historia contenida en una política. En este sentido, una política es para el gestor del sector público lo que es un prospecto para un empresario privado. Vista la transacción desde la perspectiva del gerente, este recibe una autorización para usar recursos para lograr fines públicos a través de medios concretos. Vista la transacción desde el lado del ciudadano, la autorización es la compra de una actuación agregada que promete crear valor. Es un acuerdo colectivo, político para resolver un problema (o explotar una oportunidad) de una manera particular. La política es la respuesta que una sociedad democrática liberal ha dado a la cuestión (analíticamente insoluble) de qué cosas deben ser producidas para fines colectivos con recursos públicos».

Para generar valor público los gerentes deben diseñar lo que Moor denomina «triángulo estratégico», intentando responder a tres preguntas: si su visión de los propósitos que atribuye a la organización que dirige son de valor público; si conseguirá el apoyo político y legal necesario para llevarlos a cabo; y si serán factibles desde un punto de vista administrativo y operativo. Para que su estrategia prospere necesitará integrar estos tres componentes que afectan a los aspectos sustantivos de la política, a los políticos y a los administrativos.

2 La teoría de la elección pública, especialmente de la obra de NISKANEN, Bureaucracy and representative government (1971) constituye, según AUCOIN (1990), otra de las fuentes principales de las que se nutre el Nuevo Management Público. 
Para la gestión política señala varias técnicas a las que puede recurrir el gerente público: entre ellas menciona el marketing del sector público y la comunicación estratégica. Consciente de las dudas que pueda plantear el manejo de este tipo de herramientas en el sector público señala que «lo que es estratégico de la comunicación no es que esté diseñada para manipular, sino que está pensada para hacer progresar políticas concretas o estrategias organizativas haciéndolas comprensibles y alentando el apoyo y la cooperación de aquellos que deben trabajar juntos para producir el resultado pretendido». Concibe el propio marketing como un instrumento que incrementa la rendición de cuentas hacia los consumidores. Una orientación de mercado -como es la propuesta por el marketing actual- donde los administradores investiguen a través de encuestas y grupos de discusión los atributos de los productos que demandan los consumidores y que diseñe productos para satisfacer esas demandas, lejos de ser manipulador y debilitar a los ciudadanos se convierte en una forma de reforzar la rendición de cuentas hacia los ciudadanos y sus representantes. Por otra parte, la comunicación es vital para hacer llegar la información a los ciudadanos y conseguir una movilización social para alcanzar los objetivos que persigue la organización. Obviamente, debe tratarse de una comunicación «sincera, precisa y consistente con la misión general de la organización en lugar de engañosa, falsa y manipuladora».

\subsection{Bozeman: la autoridad política de las políticas, la identificación de los valores públicos de las políticas y los fallos públicos}

Cuando Bozeman, en su libro Todas las organizaciones son públicas: tendiendo un puente entre las teorías corporativas privadas y públicas $(1987,1998)$, formuló su famosa proposición de que «algunas organizaciones son gubernamentales, pero todas las organizaciones son públicas» quiso llamar la atención sobre la existencia de dos fuentes de autoridad, la económica y la política, que influyen en el comportamiento de las organizaciones. Al mismo tiempo, quiso subrayaba la conveniencia de representar ambos tipos de autoridad como dimensiones, por lo que una organización no es totalmente «pública» o «privada», sino que es una u otra en mayor o menor grado (págs. 43-44 edición español). Asimismo, se refirió al concepto de «lo público» (publicness) para hacer referencia al grado en que una organización se ve afectada por la autoridad política.

Desde esta perspectiva, el estudio de la opinión pública sobre las políticas públicas nos informa también sobre la autoridad política, concretamente respecto de lo que el autor denomina la «autoridad política primaria», esto es, la que emana directamente de los ciudadanos. "La autoridad primaria, aunque es difícil de sondear, tiene efectos sobre las valoraciones de: la legitimidad del Estado (State); el papel y alcance del Estado (government); y las políticas. Respecto a estas últimas influye sobre las valoraciones de las macropolíticas [macropolicies] (opiniones centradas en clases generales de acciones de política [policy actions] y las de las micropolíticas [micropolicies] (opiniones de política pública concretas)» (pág. 69-71 edición inglés). Ello es relevante, entre otros motivos, porque tanto las organizaciones como las políticas se pueden ver afectadas en un grado u otro por la autoridad económica o por la autoridad política. Por regla general se da un mix de ambos tipos de autoridades. De este modo, los estudios de opinión pública podrían aproximarnos al conocimiento del grado de «publicness» de las políticas.

En un desarrollo posterior de esta misma teoría en su libro Public Values and Public Interest: Counterbalancing Economic Individualism, el autor intenta ofrecer una alternativa filosófica y práctica al individualismo económico. En el terreno práctico propone la gestión de lo público [managing publicness] como alternativa práctica a la privatización y al Nuevo Management Público. La gestión de lo público toma como punto de partida los valores públicos y el ideal del interés público es su objetivo final. Desde el punto de vista filosófico, recurre a un enfoque pragmático de la teoría del interés público - de carácter empírico y deliberativo- y a un modelo para entender el criterio de los valores públicos, un modelo para trazar los valores públicos [public value mapping mode/]) que pueda ayudar en la deliberación de las políticas. Concretamente, su modelo intenta establecer criterios para ayudar a determinar el grado en que los valores públicos están teniendo éxito o están fallando. En última instancia, el «fallo de los valores públicos» se produce «cuando ni el mercado ni el sector público proporcionan los bienes y servicios necesarios para lograr los valores públicos».

Desde esta perspectiva, identificar los valores públicos que están en juego en una política será el punto de partida, tanto para deliberar sobre cómo debe ser esta hasta para evaluarla, y los estudios de opinión pública pueden ayudar en esta tarea.

Define el término valores públicos de una sociedad como:

«aquellos que proporcionan un consenso normativo sobre (a) los derechos, beneficios y prerrogativas a los que los ciudadanos deberían (y a los que no deberían) tener derecho; (B) las 
obligaciones de los ciudadanos con la sociedad, el Estado y entre sí; y c) los principios sobre los que deben basarse los gobiernos y las políticas».

Distingue entre valores públicos y valores públicos individuales. Estos últimos:

«son las preferencias concretas de cada individuo en lo que respecta, por una parte, a los derechos, obligaciones y beneficios a los que tienen derecho los ciudadanos y, por otra, las obligaciones que se esperan de los ciudadanos y sus representantes designados».

Los valores individuales son detectables a través de encuestas y otros instrumentos de revelación de preferencias, como las elecciones o los comportamientos de los consumidores. Más problemático resulta identificar los valores públicos de una sociedad y, aún en mayor medida, desarrollar acciones concretas -en particular políticas- que permitan alcanzarlos.

En cuanto a los valores públicos y la opinión pública, señala lo siguiente:

«¿Cómo difieren los valores públicos de la opinión pública? La línea no siempre es clara, porque las opiniones públicas son a menudo opiniones sobre los valores públicos. Pero la distinción es de duración temporal. Mientras que la opinión pública es altamente volátil, tanto en sus preocupaciones como en sus direcciones, los valores públicos son mucho más estables. Pueden entrar nuevos valores públicos y los viejos pueden salir, pero generalmente sólo después de un gran cambio social y el paso de generaciones.».

La identificación de los valores públicos se convierte así en una vara de medir con la que evaluar los fallos públicos y el horizonte normativo que guíe a los directivos públicos.

\section{CONSIDERACIONES NORMATIVAS SOBRE LA RELACIÓN ENTRE OPINIÓN PÚBLICA Y ADMINISTRACIÓN PÚBLICA}

De acuerdo con el plan de exposición esbozado en la introducción corresponde ahora juntar las piezas y construir el mosaico. Desde un punto de vista normativo hacemos nuestra la idea de Lasswell de que en una democracia la relación entre opinión pública y política pública es bidireccional: el gobierno actúa sobre la opinión y esta debe hacerlo a su vez sobre el gobierno, estableciéndose un intercambio abierto entre opinión y política pública. A partir de aquí cabe plantearse dos preguntas: ¿cuál debe ser el alcance de la influencia de la opinión pública sobre la administración y las políticas públicas? Y ¿hasta dónde es legítimo que el gobierno actúe sobre la opinión pública?

\section{1. ¿Cuál debe ser el alcance de la influencia de la opinión pública sobre la administración y las políticas públicas?}

Respecto a la primera pregunta ¿hasta dónde debe influir la opinión pública sobre la administración y las políticas públicas? la teoría ha ofrecido diferentes respuestas que podrían ordenarse en una escala de menor a mayor influencia.

El primer peldaño de esa escala, el más elemental de todos y no por ello menos relevante, nos lo proporcionaría Wilson al atribuir a la opinión pública un papel de «crítica autorizada» que controla las grandes fuerzas de la formación de las políticas. Desde esta perspectiva, la opinión pública bien pudiera proporcionar un marco, más o menos difuso, dentro del cual debieran desenvolverse las políticas, aunque en ocasiones quienes adoptan decisiones opten por elaborar políticas fuera de los límites de ese marco, ya sea porque sacrifiquen la receptividad ante la responsabilidad y compromiso con los valores públicos y el interés general o por otros motivos.

Un segundo peldaño, atribuiría a la opinión pública un papel más activo en el que la administración escucharía a los ciudadanos como parte de su proceso de definición de estrategias y planificación, recurriendo entre otros instrumentos a técnicas de investigación de mercados. Así, si retomamos las ideas de Simon, Smithburg y Thomson los sondeos de opinión serían una herramienta administrativa que nos proporcionan información de inteligencia para planificar políticas, ajustar regulaciones y diseñar servicios. Moore, en esta misma línea, recomienda hacer uso del marketing para diseñar servicios e incluso la investigación de mercados puede servir también para indagar los propósitos de las políticas y los valores públicos a promocionar en 
última instancia, en torno a los cuales los gerentes añadan valor público. Lasswell nos recuerda también que los expertos en opinión pública y comunicación a través de sus investigaciones pueden aportar información valiosa en todas las etapas del ciclo de las políticas públicas.

En el último peldaño, los ciudadanos adquieren un control mayor de la situación respecto a la administración mediante diferentes vías: ya sea porque, como recomienda la Nueva Administración Pública, participen directamente en los procesos de adopción de decisiones que les afectan; o bien, como propondría la teoría de la Elección Pública, porque se diseñen mecanismos institucionales de provisión y producción de servicios que permitan a los ciudadanos votar con los pies, esto es, elegir paquetes de servicios e impuestos que se ajusten mejor a sus preferencias individuales; finalmente, como sugiere esta misma teoría o la de la Nueva Gerencia Pública, porque cuando sea posible cada individuo pueda elegir su productor de servicios públicos.

Obviamente, estos escalones no son incompatibles entre sí y pueden recorrerse todos ellos. De hecho, forman parte de la práctica cotidiana de las administraciones públicas, aunque bien es verdad que las posibilidades técnicas de aplicación de cada uno de ellos varía en función del nivel de gobierno, el área de política pública y el tipo de bien o servicio. Asimismo, pueden llevar asociados concepciones distintas de la democracia y de la administración democrática.

\section{2. ¿Hasta dónde es legítimo que el gobierno actúe sobre la opinión pública?}

La segunda pregunta que nos planteábamos era la de ¿hasta dónde es legítimo que el gobierno actúe sobre la opinión pública? Las teorías que hemos repasado y los propios comportamientos de las administraciones nos pueden ayudar a contestar esta cuestión.

\subsubsection{La interpretación y defensa de las políticas}

La función de interpretar y defender las políticas públicas a la que alude White es aceptada, al menos cuando la llevan a cabo los miembros del gobierno y del área de dirección política de los distintos departamentos del poder ejecutivo en determinados foros. Este sería el caso de los marcos institucionales de rendición de cuentas, deliberación y decisión -en particular los parlamentos-; también en las comparecencias ante los medios de comunicación o en actos recogidos por estos; en los órganos en los que participan los grupos de interés y en las reuniones informales con estos; y durante las campañas electorales.

La «defensa de la política» (policy advocacy) entendida como «la investigación y búsqueda de argumentos con el propósito de influir en la agenda de las políticas ya sea desde dentro o desde fuera del gobierno» (Parsons, 1995) es una de las formas que adquiere el análisis de políticas aplicado por los analistas que trabajan para los gobiernos o para los grupos de presión. De hecho, la argumentación y la comunicación de las políticas forma parte del proceso de elaboración y desarrollo de las políticas -ya sea de forma prospectiva o retrospectiva respecto de la decisión-, y por medio de ellas se buscan argumentos con los que participar en los procesos de deliberación, persuadir a los actores y, en su caso, a la opinión pública, propiciando el consenso, el apoyo político a la política o, al menos, intentando aminorar resistencias para hacer viable políticamente la política pública (Majone, 1989); hasta el punto de que el análisis de las políticas y su argumentación se convierte en un poderoso recurso en la lucha política en torno a las políticas. El enfoque de la construcción social de las políticas ha destacado en qué medida el diseño de la política y la forma en la que se delimitan los públicos objetivos de las políticas pueden ayudar a conformar la opinión pública (Schneider y otros, 2014). En enfoques más recientes, como los desarrollados desde el marco de la Narrativa de las Políticas, se abunda en esta misma línea intentando descomponer la estructura narrativa de los relatos de las políticas y las estrategias de comunicación a las que recurren las coaliciones promotoras y opositoras de ellas, con objeto de ganarse el apoyo de la opinión pública y así favorecer o dificultar el cambio de las políticas (Jones y otros, 2014).

\subsubsection{La gestión de la opinión pública mediante recursos públicos}

Todas estas actividades son consideradas legítimas; más problemático puede resultar, en cambio, el gestionar la opinión mediante recursos públicos. En la gestión de la opinión cabe distinguir dos tipos de actividades: conocer el estado de la opinión y cultivar una opinión pública favorable. 


\subsubsection{Conocer el estado de la opinión}

La primera de estas actividades, conocer el estado de la opinión, no debiera plantear objeciones. De hecho, algunos gobiernos crean organismos especializados para ello, como pueda ser en España el Centro de Investigaciones Sociológicas, o existen unidades organizativas que recaban sondeos de opinión para informar sobre el estado de la opinión a los líderes de los ejecutivos, como los Eurobarómetros encargados por la Dirección General de Comunicación de la Comisión Europea. Lo importante es la transparencia con la que se lleve a cabo esta actividad. En el caso de las encuestas que se financian con fondos públicos es razonable esperar que los datos obtenidos tengan una amplia difusión. En los sistemas más avanzados la información es accesible, estando a disposición del público en plazos breves desde que han sido recabados y depurados los datos, de forma gratuita o a precios asequibles y en formatos que permiten consultar tanto la información agregada como los microdatos. Ello no obsta para que puedan existir periodos de embargo de la información sin poner a disposición del público algunas variables o el conjunto del estudio durante un período razonable.

Las encuestas, con ser importantes, no son las únicas herramientas a las que recurren los gobiernos para conocer el estado de la opinión pública. Es frecuente que las administraciones públicas hagan uso de diversas técnicas de investigación de mercados como puedan ser: los buzones de quejas y sugerencias, los grupos de discusión, los paneles de encuestas, las entrevistas en profundidad, la observación directa, la investigación etnográfica, el comprador misterioso, el análisis de contenido de los medios de comunicación y de los documentos de los grupos de interés y otros actores involucrados en las políticas, el análisis de datos del comportamiento de los ciudadanos en relación con políticas y servicios o la investigación experimental. Cualquier técnica de investigación de mercados sirve para "escuchar» a los ciudadanos y puede ser considerada a su vez como un instrumento de participación ciudadana.

Por otra parte, cualquier instrumento de participación puede servir como una herramienta de investigación de mercados. Así sucede con los mecanismos de información a los ciudadanos que emplean las administraciones públicas, como los periodos de alegaciones a documentos o planes expuestos al público, las exposiciones en las que se muestran esos mismos planes en formatos asequibles, o los ruegos y preguntas al final de las reuniones de los órganos de gobierno que se llevan a cabo en el ámbito local. Asimismo, son de utilidad para conocer la opinión los referenda y las consultas ciudadanas en las que los ciudadanos expresan sus preferencias sobre temas concretos. Finalmente, los espacios de intercambio entre la administración y los ciudadanos, como los foros o consejos sectoriales y territoriales, las webs interactivas, los ejercicios de visión de futuro o los jurados ciudadanos, son herramientas de participación que ayudan a sondear la opinión.

Las redes de oficinas y sistemas de información directa al público, ya sean en formato físico o telemático, no solo cumplen una función de información y promoción directa de los servicios públicos, sino que también pueden ser aprovechados por el contacto entre los empleados públicos que los atienden y los ciudadanos para recabar información de inteligencia que ayude a diseñar políticas y servicios. Los programas piloto, y cualquier tipo de prueba o incentivo utilizado por el sector público, permite escuchar a los ciudadanos y llevar a cabo una «prueba de mercado» de políticas y servicios públicos. La figura del defensor del pueblo o de los usuarios de los servicios es también una fuente importante para conocer las demandas ciudadanas.

Más recientemente, la observación de las redes sociales junto con los estudios de grandes cantidades de datos mediante las herramientas del análisis de redes nos ofrecen oportunidades adicionales para indagar la opinión pública, especialmente ante temas concretos, incluso en espacios territoriales muy delimitados y comunidades específicas de usuarios de servicios. Utilizadas con prudencia, especialmente por los sesgos que contienen las muestras, ofrecen oportunidades adicionales de indagación en tiempo real de la opinión pública sobre políticas y servicios.

En definitiva, el dedicar recursos públicos para conocer el estado de la opinión pública -ya sea mediante encuestas o con cualquiera de los mecanismos arriba mencionados- no debería resultar objetable. De hecho, todas estas herramientas son de uso común en el sector público y ayudan a escuchar la opinión de los ciudadanos.

\subsubsection{La formación y administración de la opinión}

La gestión de la opinión no solo implica llevar a cabo una adecuada investigación de mercados, sino también cultivar una opinión pública favorable hacia las instituciones, las políticas, los programas y los ser- 
vicios públicos. Es en este terreno donde entra de lleno el marketing público y las campañas de promoción y comunicación institucional, incluyendo las que recurren a la publicidad.

Una adecuada estrategia de marketing sin duda contribuye a generar una opinión pública favorable. Ahora bien, ¿hasta qué punto es legítimo el uso del marketing en el sector público? Nadie discute la pertinencia del marketing político que desarrollan los partidos, pagado con sus propios fondos, con objeto de incidir en el comportamiento electoral. Sin embargo, puede resultar más dudosa su aplicación por parte del sector público una vez que se ha celebrado la fiesta de la noche electoral y llega la hora de gobernar.

El concepto de marketing tiene un perfil de imagen muy preciso. Con frecuencia se asocia a la venta y a intensas campañas de publicidad que tienen como propósito estimular el consumo e incluso la manipulación deliberada de la demanda. En este sentido, la aplicación de las herramientas de marketing al sector público, pagadas además con fondos públicos, sería inapropiada cuando no éticamente dudosa y poco profesional, no teniendo otro sentido que el de intentar manipular a la opinión pública con objeto de obtener un buen rendimiento electoral del partido o la coalición que se encuentre en el gobierno. Ahora bien, este perfil de imagen, que se ajusta muy bien a las organizaciones orientadas hacia la venta, poco tiene que ver con una concepción más avanzada del marketing.

El marketing puede ser considerado como la suma de una filosofía más un conjunto de herramientas para implantar esa filosofía. La filosofía podría sintetizarse en la idea de que la justificación de cualquier organización es satisfacer las necesidades de sus clientes (Crompton y Lamb, 1986). Si sustituimos la palabra organización por administración y la de clientes por ciudadanos -la justificación de cualquier administración es la satisfacción de las necesidades de los ciudadanos- obtenemos un concepto muy cercano al de la administración receptiva, esto es, la de una administración que da respuesta e incluso se anticipa a las necesidades y demandas de los ciudadanos. Una organización orientada hacia el marketing -o si se prefiere, una administración orientada hacia el mercado- lo primero que hace es indagar las necesidades de los distintos grupos de clientes -o segmentos de mercado-. Posteriormente, desarrolla programas o servicios que satisfagan las necesidades identificadas en la etapa anterior. Finalmente, hace un esfuerzo de promoción para informar a sus clientes de la existencia de dichos programas con objeto de que los utilicen. Una administración que opere con esa filosofía y que sea capaz de implementarla conseguirá no solo ser más eficiente, sino obtener notables niveles de apoyo de los públicos a los que se dirija. Por otra parte, nada cabe objetar a este modo de proceder pues, lejos de entrar en contradicción con los valores públicos, contribuye a reafirmarlos.

Para implantar esta filosofía se hace necesario desarrollar una serie de actividades de marketing. «El marketing consiste en el análisis, la planificación, la realización y el control de programas cuidadosamente formulados con objeto de efectuar intercambios voluntarios de valor con mercados-objetivo». Ello significa, sobre todo, «adecuar la oferta de la organización a las necesidades y deseos de los mercados-objetivo, y al uso eficaz de las técnicas de determinación de precio, de la comunicación y de la distribución para informar, motivar y servir al mercado» (Kotler y Lee, 2006).

El concepto de intercambio resulta central para el marketing. Desde esta perspectiva, el propósito del marketing público consiste en facilitar el intercambio entre la Administración y los ciudadanos. En este sentido, la Administración elabora programas que satisfacen las necesidades materiales y simbólicas de los ciudadanos y a cambio la Administración obtiene de los ciudadanos su apoyo. Este se expresa de diversas maneras: a través de impuestos y otros instrumentos financieros, como tasas o precios públicos que el ciudadano paga directamente a la administración; aportando diversos costes económicos para el ciudadano que son necesarios para la utilización del servicio o el cumplimiento de la regulación y que no percibe la administración -por ejemplo costes de desplazamiento hasta el punto donde se ofrece el servicio- y otros costes no monetarios -tiempo, energía, costes sicológicos, etc.- necesarios para consumir el servicio o cumplir la regulación. Asimismo, existen otras formas de expresión del apoyo que no implican costes para el ciudadano, como pueden ser determinados comportamientos observables -el consumo de servicios públicos o el cumplimiento de las regulaciones- 0 a través de la expresión de actitudes o predisposiciones para actuar a favor de algo o alguien. Los sondeos de opinión nos ayudan a determinar en qué medida existen o no esas actitudes favorables, así como lo hacen también muchas de las formas de participación política, tanto convencionales como no convencionales.

Para facilitar el intercambio entre la Administración y el ciudadano, una administración orientada hacia el mercado desarrollará diversas actividades de marketing: recabará información de marketing (la investigación de mercados tiene este propósito); establecerá la misión de la organización; diseñará estrategias de marketing; las implementará y las evaluará. La estrategia de marketing consistirá en el establecimiento de objetivos, la selección de públicos y segmentos de mercado que se quiera atender -incluida la asignación 
de recursos para cada segmento- y la elaboración del mix o combinado de marketing. Este último consistirá en tomar decisiones relativas a los programas que se proporcionarán, su distribución en el territorio y el tiempo, el precio monetario y no monetario que los ciudadanos deberán pagar y el sistema de promoción y comunicación más adecuado (Crompton y Lamb, 1986).

Si se observa con detenimiento esta relación de actividades de marketing, conciernen todas ellas a cuestiones sobre las que los gobiernos y administraciones deciden cotidianamente y nada hay que objetar. La peculiaridad del marketing es que las impregna de una filosofía -la orientación hacia mercado- y las planifica conjuntamente aprovechando las sinergias entre todas ellas ${ }^{3}$.

No obstante, dentro del mix de marketing hay un punto, el de la promoción y la comunicación institucional, sobre el que puedan plantearse objeciones, especialmente cuando se recurre a campañas publicitarias. Veamos con detenimiento esta cuestión.

Las campañas de promoción y comunicación institucional pueden recurrir a uno o varios instrumentos de comunicación: la publicidad, la información directa, las pruebas o incentivos, la información de servicio público, la publicidad no pagada ${ }^{4}$, las relaciones con los medios de comunicación y la participación en redes sociales ${ }^{5}$. A todas estas herramientas recurre también la administración y, en general, la única cuestión sobre la que se plantean objeciones es cuando se emprenden campañas publicitarias con fondos públicos, el resto de elementos del mix de promoción suelen considerarse legítimos.

Pero incluso la publicidad financiada con fondos públicos puede ser aceptada en determinadas circunstancias. Desde la óptica del marketing la promoción es básicamente un ejercicio de comunicación. Consiste en facilitar los intercambios con los clientes actuales o potenciales mediante la información, la educación, la persuasión, y recordando los beneficios ofrecidos por la administración, sus programas o servicios (Crompton y Lamb, 1986). Los ciudadanos necesitan información básica sobre los servicios y regulaciones, incluidos datos de fechas, lugares en los que se pueda acceder a los servicios, importes de precios o tasas que deben pagar, etc. Asimismo, la administración con frecuencia debe desarrollar una labor pedagógica mostrando a futuros usuarios el valor de sus programas y servicios; e incluso para determinadas cuestiones de interés social, intentará incidir en sus actitudes, creencias y valores para intentar influir en sus comportamientos posteriores. También deberá persuadir a los ciudadanos para que acudan a los servicios, cumplan las regulaciones o modifiquen comportamientos en su propio beneficio y/o en el del interés general. Finalmente, a aquellas personas que ya son consumidores de programas o servicios, o que cumplen las regulaciones, habrá que recordarles los beneficios que obtienen de ello para que en su caso lo sigan haciendo. Ninguna de estas metas es, en principio, objetable y de hecho observamos como la administración las procura de forma activa en multitud de áreas de política pública y de servicios públicos. Más bien al contrario, si la administración no cuenta con una promoción y comunicación adecuadas los servicios y políticas públicas no funcionarán eficientemente y el cumplimiento de las regulaciones se resentirá.

Caso distinto es interpretar que las campañas han sido diseñadas para otros propósitos, como pueda ser la promoción de las autoridades en ejercicio en lugar de las instituciones que dirigen -lo que coloquialmente se denomina «autobombo»-, o el intento de influir en el comportamiento electoral, en particular cuando se llevan a cabo en periodos cercanos a la campaña electoral. La promoción de políticas suele ser más problemática que la promoción de servicios y las campañas que intentan promover un cambio social sobre los valores y comportamientos pueden ser también cuestionadas en la medida en que no exista una amplio consenso social y político sobre ellos. Obviamente, aquellas campañas que no son rigurosas en cuanto a la veracidad de la información que trasmiten, engañosas y manipuladoras son poco tolerables en general y más cuando se llevan a cabo con fondos públicos. Hay también aspectos formales que se deben cuidar,

3 La administración por lo común opera como una suerte de déspota ilustrado manteniendo una orientación hacia la producción -desarrolla los servicios o programas que considera oportunos, esperando que estos satisfagan las necesidades de los clientes- o hacia la venta -desarrolla sus programas y hace un intenso esfuerzo de promoción esperando que sus servicios satisfagan las necesidades de sus clientes-, y no hacia el mercado -mediante la identificación de necesidades consultando a los propios ciudadanos, con el desarrollo de programas ajustados a esas necesidades e informando de la existencia de esos programas-. Asimismo, lejos de planificar de forma integrada el conjunto de las actividades de marketing, las gestiona todas ellas pero de manera inconexa.

4 La publicidad no pagada son noticias o comentarios editoriales sobre una organización y sus programas que se transmiten a través de medios de comunicación de masas sin cobro alguno para la administración.

5 Todas ellas, excepto la última, implican una forma de comunicación unidireccional que va de la administración al ciudadano -que puede estar mediada o no por los medios de comunicación de masas-. En cambio, la participación en redes sociales implica una comunicación de carácter bidireccional y requiere para gestionarla adecuadamente un importante esfuerzo por parte de la administración. 
como son la pertinencia institucional de los contenidos o el evitar la aparición de autoridades en ejercicio en los materiales impresos o audiovisuales de las campañas.

En definitiva, con sus limitaciones, las campañas de publicidad disponen de una amplio espacio mediante el que intentar gestionar la opinión pública con fondos públicos de una manera legítima.

Finalmente, hay una cuestión más desde el punto de vista normativo que merece ser tomada en consideración: se refiere a quién compete la interpretación y defensa de las políticas y la gestión de la opinión mediante recursos públicos, tanto en lo que respecta a conocer el estado de la opinión como a la formación y administración de la opinión. Como vimos más arriba, para White esta era una función propia del nivel político, en particular de los líderes del ejecutivo, de los responsables de los departamentos y sus gabinetes políticos, «resultando particularmente inadecuada en el caso de los funcionarios de carrera» (White, 1926, 1955). Desde luego la esfera política está claramente involucrada en estas cuestiones; ahora bien, tanto el tamaño del sector público, como su alcance funcional y la complejidad y especialización técnica que requieren estas tareas en el siglo XXI poco tiene que ver con los de la época en que White escribió la primera edición de su manual. Hoy en día cualquier directivo público se encuentra involucrado en estas cuestiones y cuenta para ello con el apoyo de estructuras organizativas, empleados públicos, investigadores y empresas especializadas en conocer la opinión e intentar cultivar una opinión pública favorable.

\section{CONCLUSIONES}

La cuestión de la opinión pública es un asunto que ha recibido la atención de los autores clásicos de la Ciencia de la Administración debido, entre otros motivos, a los papeles que cabe atribuir a la opinión en la Administración Pública de un sistema político democrático. En la medida en que la deferencia hacia la Administración y otras instituciones jerárquicas vaya en descenso, la competencia democrática de la ciudadanía continúe creciendo y la democracia representativa se vea complementada con fórmulas de democracia directa que supongan un incremento de la participación de los ciudadanos, se hará cada vez más necesario volver a reflexionar sobre la opinión pública desde una perspectiva administrativa. No solo en lo que atañe a su papel en el cambio profundo de las políticas que de cuando en cuando tiene lugar, sino también en otros procesos administrativos más comunes y cotidianos, incluida la planificación y gestión de las organizaciones y de los servicios públicos, o su participación directa mediante diferentes instrumentos de participación ciudadana.

Es importante destacar que la relación entre opinión pública y Administración es de intercambio, de influencia de la opinión sobre la Administración y de influencia también de la Administración sobre la opinión. La Administración no solo debe estar atenta a las demandas de los ciudadanos y ser receptiva a ellas, sino que también debe gestionar la opinión de forma responsable, promocionando con ello los valores públicos y el interés general. Los autores que hemos revisado aluden a ambos lados de la ecuación con lo que ello comporta del uso de múltiples herramientas para conocer la opinión pública e intentar influir sobre ella, de las que hemos hecho mención y que forman parte el repertorio cotidiano de instrumentos de gestión pública.

Desde una perspectiva convencional puede pensarse que el conocimiento y la gestión de la opinión pública son tareas que competen a la esfera política y que los empleados públicos profesionales deberían estar alejados de esas actividades. Si se mantiene una rígida separación entre política y administración y entre la formulación de las políticas y su implementación, debería de ser así. Sin embargo, la función ejecutiva actual poco entiende de rígidas separaciones entre política y administración o entre formulación e implementación de políticas, de modo que los directivos públicos, sean estos de nombramiento político o funcionarios profesionales, con frecuencia están directamente involucrados en escuchar y gestionar la opinión. Por otro lado, el sondeo de la opinión y la gestión de esta se han vuelto tan complejos que requieren de unidades organizativas especializadas y de empleados públicos profesionales, cualificados en estos campos para atenderlas.

Al margen de las consideraciones normativas, la propia observación del comportamiento de las organizaciones públicas nos muestra como la opinión pública desempeña numerosos papeles no excluyentes entre sí, desde ejercer la crítica autorizada y el establecimiento de un marco dentro del cual se lleve a cabo la formación de las políticas, hasta otras más avanzadas que incluyan la participación directa de los ciudadanos, pasando por tener en cuenta la opinión pública en los procesos de definición de estrategias y la planificación administrativa. Además, observamos los importantes esfuerzos que mediante diversos mecanismos realizan los directivos públicos, tanto políticos como funcionarios, por conocer y tener en cuanta a la opinión pública; y como, al mismo tiempo, se dirigen a ella para informarla, intentar conformarla y hacer más eficaz y responsable la gestión de políticas y servicios públicos. 


\section{REFERENCIAS BIBLIOGRÁFICAS}

AUCOIN, P. (1990): "Administrative reform in public management: paradigms, principles, paradoxes and pendulums", en Governance, núm. 3, págs. 115-137. DOI: https://doi.org/10.1111/j.1468-0491.1990.tb00111.x.

BERRET, E. J. (1996): "The Role of Public Opinion in Public Administration", en The Annals of the American Academy of Political and Social Science, núm. 537, págs. 150-162.

BOZEMAN, B. (1998): Todas las organizaciones son públicas: tendiendo un puente entre las teorías corporativas privadas y públicas. México, D. F.: Fondo de Cultura Económica. Edición en inglés de 1987.

BOZEMAN, B. (2007): Public Values and Public Interest: Counterbalancing Economic Individualism. Washington D. C.: Georgetown University Press.

FREDERICKSON, H. G. (1980): New Public Administration. Alabama: The University of Alabama Press.

CROMPTON, J. L. y LAMB, C. W. (1986): Marketing Government and Social Services. Nueva York: John Wiley.

GOODNOW, F. J. (1900): Politics and Administration. A Study in Government. Nueva York: Macmillan.

HOOD, C. (2002): "New Public Management", en International Encyclopedia of the Social \& Behavioral Sciences, págs. 12.553-12.556. Elsevier. DOI: https://doi.org/10.1016/b0-08-043076-7/01180-3.

JONES, M. D., SHANAHAN, E. A. y MCBETH, M. K. (2014): The Science of Stories: Applications of the Narrative Policy Framework. New York: Palgrave. DOI: https://doi.org/10.1057/9781137485861.

KOTLER, P. y LEE, N. (2006): Marketing in the Public Sector: A Roadmap for Improved Performance. Upper Saddle River: Wharton School Publishing.

LASSWELL, H. D. (1941): Democracy Through Public Opinion. Menasha: George Banta.

LIPPMAN, W. (1922): Public Opinion. Project Gutenberg EBook. 2014.

MAJONE, G. (1989): Evidence, Argument, and Persuasion in the Policy Process. New Haven: Yale University Press.

MOORE, M. H. (1995): Creating Public Value: Strategic Management in Government. Cambridge: Harvard University Press.

NISKANEN, W. A. (1971): Bureaucracy and representative government. Chicago: Aldine-Atherton. DOI: https://doi. org/10.4324/9781315081878.

OSBORNE, D. y GAEBLER, T. (1992): Reinventing Government: How the Entrepreneurial Spirit is Transforming the Public Sector. Reading: Addison-Wesley.

OSTROM, V. (1989): The Intellectual Crisis in American Public Administration. Tuscaloosa: University of Alabama Press, 2. ${ }^{a}$ ed. La primera edición es de 1971.

PARSONS, W. (1995): Public policy: an introduction to the theory and practice of policy analysis. Cheltenham: Edward Elgar.

PETERS, T. J. y WATERMAN, R. H. (1992): En busca de la excelencia. Lecciones de las empresas mejor gestionadas de Estados Unidos. Barcelona: Ediciones Folio. Edición en inglés de 1982.

POLLITT, C. (2003): The Essential Public Manager. Philadelphia: Open University Press.

SCHNEIDER, A. L., INGRAM, H. y DELEON, P. (2014): "Democratic Policy Design: Social Construction of Target Populations", en SABATIER, P. A. y WEIBLE, C. M. (eds.): Theory of the Policy Process. Boulder: Westview Press, 3. ${ }^{\mathrm{a}}$ ed.

SIMON, H. A., SMITHBURG, D. W. y THOMSON, V. A. (1950): Public Administration. Nueva York: Alfred A. Knopf.

WHITE, L. D. (1995): Introduction to the Study of Public Administration. Nueva York: Macmillan, 4. ${ }^{a}$ ed. La primera edición es de 1926 .

WILSON, W. (1887): “The Study of Administration”, en Political Science Quarterly, vol. 2, núm. 2, págs. 197-222. DOI: https://doi.org/10.2307/2139277. 